\title{
BREAST CANCER IN AN 18-YEAR-OLD PATIENT: A CASE REPORT
}

Maria Letícia Moraes¹, Eduardo Resende Sousa e Silva', Laura Viana de Lima¹, Carla Paulinelli Seba', Isabela Vieira Bastos ${ }^{1}$, Ana Paula Teixeira Leite'1, João Emanuel Meireles Gonçalves', Renata Pereira Fontoura'

${ }^{1}$ Brasília (DF) - Brazil.

Objective: The present report aims to describe a case of invasive ductal carcinoma in a young patient. Methodology: Active search in medical records. Case: B.S.M., female, 18 years old, arrived at the oncology service with diagnosed ductal infiltrative carcinoma, with a tumor measuring 6 x $8 \mathrm{~cm}$, staging T4 Nx M1, with metastasis to lungs, and marker level CA 15-3 was 9,2, 4 months ago. The histopathology presented the carcinoma was moderately differentiated with intraductal component associated and the immunohistochemistry shows $95 \%$ for both estrogen and progesterone receptors, negative for HER-2 and level of $20 \%$ for Ki-67, both of them from 5 months ago. The thoracic CT from 4 months ago showed many non-calcified nodules sparse in pulmonary parenchyma up to $14 \mathrm{~mm}$, compatible with implants, increased density in retroareolar region measuring 39 x $34 \mathrm{~mm}$ with slight thickening of adjacent skin, two nodules of $5 \mathrm{~mm}$ each and axilary limph nodes up to $11 \mathrm{~mm}$. At physical examination, the tumor was compromising $70 \%$ of the right breast, which was presenting nipple retraction and ulceration. Palliative chemotherapy was initiated with Fluoracil, Doxorubicin and Cyclophosphamide (FAC) once 4 months ago (11/21/17), that was switched to Doxorubicin, Cyclphosphamide and Paclitaxel (AC-T) for unavailability of Fluoracil at the Institution. The patient was submitted to 3 sessions of FAC 3 months ago (01/22/18). At 02/16/18, at physical examination right breast presented a tumor sized 7 × 6 with nipple ulceration, demonstrating fail of treatment, and then was administered the first cycle of Docexatel. At physical examination at 03/09/18, right breast presented a tumor of $5 \times 5 \mathrm{~cm}$ with nipple injure on healing phase and the patient was conducted to the second cycle of Docexatel. Considerations: Breast cancer is considered rare before the age of 35, and the risk of having it grows rapidly with age, with its apex appearing between 40 and 60 years. The patient of the case for being 18 years of age demonstrates an exception to the age values in the statistical data. Thus, new studies are needed to understand the appearance of breast cancer in young patients. 